\title{
A multi-domain modelling and verification procedure within MBSE approach to design propulsion systems for road electric vehicles
}

\author{
Clemente Capasso $^{1}$, Moncef Hammadi $^{2}$, Stanislao Patalano $^{3, a}$, Ruixian Renaud ${ }^{2}$ \\ AND OTTORINO VEnERI ${ }^{1}$ \\ 1 Istituto Motori, CNR National Research Council of Italy, Via Marconi 4, 80125 Naples, Italy \\ 2 SUPMECA, Higher Institute of Mechanical Engineering, Paris 3 rue Fernand Hainaut, 93407 Saint-Ouen, France \\ 3 University of Naples Federico II, Department of Industrial Engineering, P.le V. Tecchio 80, 80125 Naples, Italy
}

Received 31 May 2015, Accepted 18 January 2016

\begin{abstract}
The paper deals with the model based systems engineering (MBSE) approach, focused on the designing process of propulsion systems for road electric vehicles. In particular, the paper adopts multidomain Modelling, in accordance with a top-down approach. The process, in fact, starts from the main requirement analysis of the road electric vehicle which is considered as reference. Then, a wide range of parameters, related to the characteristics of propulsion system components and resistance forces, are evaluated to build a parametric model of the propulsion system running on a road. In this way, a procedure for the evaluation of vehicle performance is accomplished within the developed simulation environment. Therefore, the procedure allows all the requirements to be satisfied, under different operative conditions, through an iterative procedure of verification for the imposed parameters. The tested operative conditions are represented in this paper by standard driving cycles, expressed in terms of vehicle speed and autonomy requirement.
\end{abstract}

Key words: Systems engineering / MBSE approach / object-oriented modelling and simulation / electric vehicles

\section{Introduction}

Systems engineering (SE) is an interdisciplinary approach, aimed to enable the realization of successful systems. It focuses on the interaction between the most relevant stakeholders and actors throughout the system's life cycle [1]. Actually, designing activities related to complex systems as well as research activities adopt the SE approach. Besides, during last years the approach has been also extended to the whole product development lifecycle covering early factors that affect the entire product lifecycle [2]. Gradually, SE was also joined to a model based (MB) approach to accomplish a unique and integrated methodology, in order to obtain modelling and simulation integration, during the whole design process [3]. Therefore, model-based systems engineering (MBSE) is expected to replace the document-centric approach that has been practiced by systems engineers in the past. Mechatronic systems are complex systems that could be rightly developed by using the MBSE approach, because they deal with multi-domain and related interactions of effects

\footnotetext{
a Corresponding author: patalano@unina.it
}

due to different existing domains [4]. Several and different contributions are known in literature and a significant part of them affects both the process-based problems and design data-related problems [5]. In reference [6], authors proposed the use of hierarchical models to support the whole design process of mechatronic systems. In reference [7], a multidisciplinary approach using SysML language and aimed to the designing of mechatronic systems is presented. On the other side, along the design datarelated problems, the authors in reference [8] present different architectural designs in the context of automation systems domain, for an efficient and effective integration solution related to data model design.

Even if the process-based problems and design datarelated problems are significant and represent urgent challenges in the designing of mechatronic systems, the MBSE approach is needed for adequate and deep methodologies and tools within the "decomposition and definition" axis, especially for "high-level design" and "detailed design" steps. In such steps, in fact, methodologies and tools are expected to realise the integrated modelling and simulations between different domains $[9,10]$. 
In reference [11], a co-modelling methodology and co-simulation tools integration framework are presented to improve the co-design process of mechatronic devices by maintaining the domain specific properties of the model components. Furthermore, the implementation of the MBSE approach within the commercial CAD/PDM environment CATIAV6, by Dassault Systemes could be considered the method known as RFLP (Requirement/Functional/Logical/Physical) [5]. In particular, such method includes multi-domain modelling within Modelica language and, therefore the related software tool is effective in providing integrated-domain design.

During the application of a V-cycle approach, the first main objective is that the system level requirements are propagated to component design level in a top-down way, allowing traceability between the models developed with different levels of abstraction. The second objective is that the system performance validation is performed by increasing the integration level according to a bottom-up way, i.e. by integrating component level behaviour in order to accomplish the whole system behaviour. This necessitates to couple multi-physics models between each other and also with control models, to be able to validate the overall system requirements.

The present paper is an extended version of a work presented in Multiphysics Modelling and Simulation for systems design and monitoring (MMSD) conference [14]. The previous work was limited to a general description of the RFLP approach and only the logical phase was detailed in the test-case. Actually, the present work will deepen the integrated multi-domain modelling, aimed to the design of the propulsion systems for road electric vehicles, accomplishing the successive definition and verification loop within a MBSE-V cycle approach. Moreover, new simulation results concerning the storage system and the electric machine are added in the present work. The paper is arranged as follows: Section 2 presents the RFLP approach. Section 3 illustrates the requirement sets, the functional analysis and the logical modelling related to the designing of power-trains for urban electric busses. Section 4 states simulation results and, finally, Section 5 draws the conclusions.

\section{RFLP approach as derived method for MBSE}

RFLP stands for Requirements engineering, Functional design, Logical design and Physical design [12] and it is a MBSE approach based on the $\mathrm{V}$-cycle design process of complex engineered systems. Figure 1 shows a description of the proposed design process.

RFLP approach helps system architects and designers in coordinating separate activities and views in a concurrent engineering platform. The RFLP approach follows a top-down decomposition process. Firstly operating on the whole complex system, then, introducing series of different sub-systems and, in the same way, the approach is applied to each sub-system to design all the system components [13]. The requirement $(\mathrm{R})$ view allows managing and verification of thousands of stakeholder needs and requirements related to different disciplines. During this phase the main features and services offered by the analysed system are defined in a specific documentation. The system architect develops the functional (F) architecture of the system in a hierarchical way, defining the functions, the links between them, the types of connections and the flows to be exchanged between functions. Logical (L) modeling is an intermediate step between functional and physical levels. Since several functions at functional level could be allocated to one technological solution, logical modelling allows system architects to define the different possible candidate solutions of the considered system. At this level, behaviour analysis can be implemented to help system architects in the choice of the best solutions. Behaviour can be implemented in logical level in two ways; either by allocating 3D Logical representation to logical components or with 0D Physical (dynamical) behaviour using Modelica language. 3D Logical representation is used to allow spatial analysis of the candidate solutions. 0D Physical modelling through Modelica language allows the analysis of the dynamic behaviour of the candidate solutions. Combining 0D Physical with 3D Logical representation helps system architects in understanding the dynamic behaviour of mechanisms and moving parts. The Physical (P) level is required for the detailed development of the components of a chosen architecture. The detailed 3-D models can therefore be developed with their parametrical relationships.

The RFLP approach allows a top-down process from Requirement to Physical. However, a bottom-up approach could also be applied. For example, a 3D logical model can be easily extracted from an existing 3D physical assembly. The 3D logical model can therefore be integrated in the logical level by using appropriate CATIA/Modelica components, which allows system architects to verify (Verification in Fig. 1) the dynamic behaviour of the system by integrating the exact inertia matrices of the assembly. This allows designers to integrate (Integration step in Fig. 1) more details in the system architecture models developed in the top-down phase. Depending on the verification results, the functional architectures could also be revised. Validation step allows designers to confirm that all the design requirements are fulfilled.

Traceability links in the RFLP approach allow designers to share modelling data and simulation results. Therefore any designer can have access to requirements and functions in link with the models he is developing. This facilitates the verification and validation steps when modifications are required.

The advantage of the MBSE-based RFLP approach, compared to classical sequential methods, is essentially the reduction of the number of modification loops during the design process of multidisciplinary systems. Indeed, with classical design methods, the multidisciplinary system design integration is carried downstream. Thus the problems of interfaces and interconnections between 


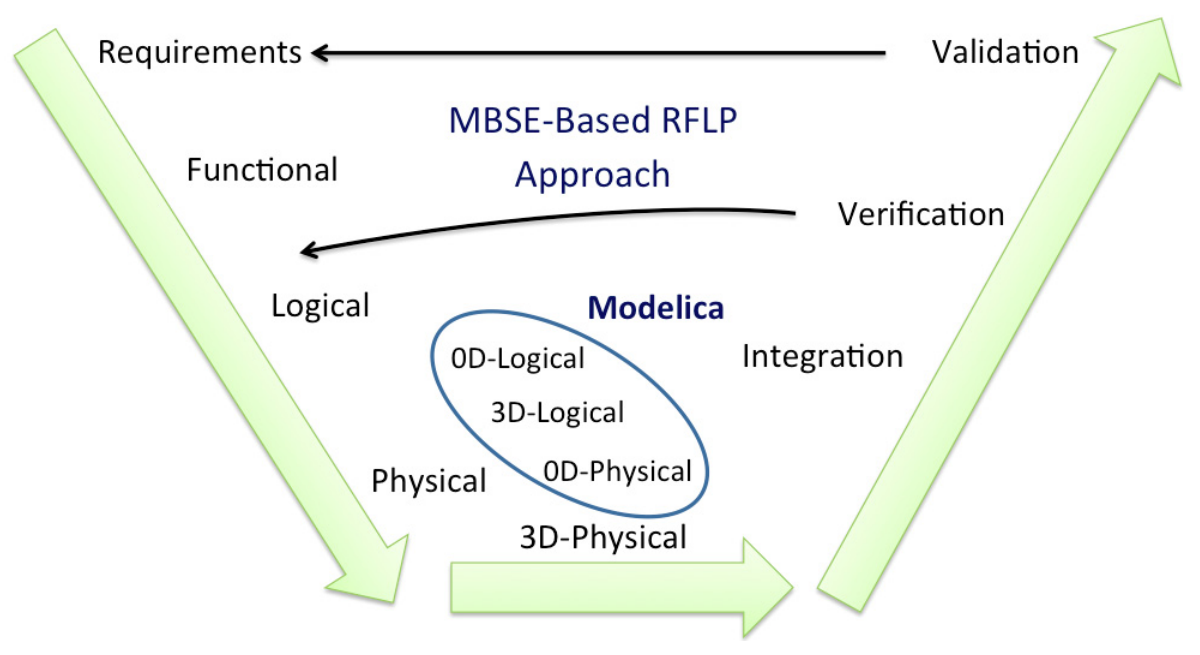

Fig. 1. Design process using MBSE-based RFLP approach.

disciplines are identified later in the design process, which imposes several modification loops, often costly. With the RFLP, however, the integration between disciples is performed in the early design process, corresponding to the RFL phases. So interfaces and interconnection constraints between disciplines are identified and taken into consideration earlier. This facilitates the integration between disciplines in the detailed design level, since integration constraints can be taken into consideration in the topdown decomposition process. Also, it facilitates the verification and the validation of the overall design, due to the traceability links established between design entities belonging to the different levels of design.

\section{Case study: designing of power-trains for urban electric busses}

The case study of an electric power-train for urban bus applications is considered in this paper. The evaluation of the main electrical and mechanical performance figures of the propulsion system supplying the vehicle on a real road is generally difficult to assess. This issue is principally related to various external factors, which depends mainly on road, traffic and environmental conditions and often are difficult to predict. For this reason a complete validation of the simulation results for the power-train can take advantage of test bench activities, conveniently carried out in research laboratories [14]. The designing of the considered propulsion system, making use of a model based approach, such as the RFLP presented in the previous section, involves many advantages, mainly in terms of time and cost reduction [15].

The main characteristics of the urban bus, which is considered as reference vehicle in this paper, are reported in Table 1.

The first step of the RFLP approach consists in the definition of the main functional requirements for the specific application under study. In this case, the first requirement is related to the need for the electric propulsion
Table 1. Main characteristics of the reference urban bus.

\begin{tabular}{cc}
\hline Weight $(\mathrm{kg})$ & 6185 \\
Main section $\left(\mathrm{m}^{2}\right)$ & 4.664 \\
Motor-Wheel transmission Ratio & $1 / 6$ \\
Aerodynamic drag coefficient & 0.7 \\
Effective rolling radius (m) & 0.4 \\
Tyres & $235 / 65 \mathrm{R} 16$ \\
Number of passengers & 18 \\
\hline
\end{tabular}

system to power the reference vehicle, whose main characteristics are reported in Table 1 . This requirement can be split in more detailed sub-requirements, which take into account that a brake bench has to represent the mechanical load of the vehicle running on a specific road, an energy storage system has to supply the electric drive with the required energy and power and a propulsion system has to follow the required driving cycle in terms of speed and torque, when running on the test bench. A further division can be performed on the base of mechanical and electrical sub-requirements. In particular, the representation of the mechanical load involves the proper evaluations of resistant forces, composed by rolling, advancing, aerodynamic and inertia forces. Moreover, the energy storage system has to ensure the autonomy and dynamic performance required by a specific mission evaluated for the reference vehicle. Finally, the proper control of resistant torque, motor torque and speed is required for the propulsion system, when running on the test-bench, to follow the driving cycle under study.

The second step of the RFLP approach, represented by the functional analysis, is obtained by means of the functional map reported in Figure 2, which is realized in CATIA V6 environment.

The above figure reports only the highest level of the functional diagram, whereas the lower levels are neglected in this figure for reasons of synthesis, although they are considered in the functional map. On the base of the scheme reported in this diagram, the propulsion system, with its auxiliary components, when running on the 


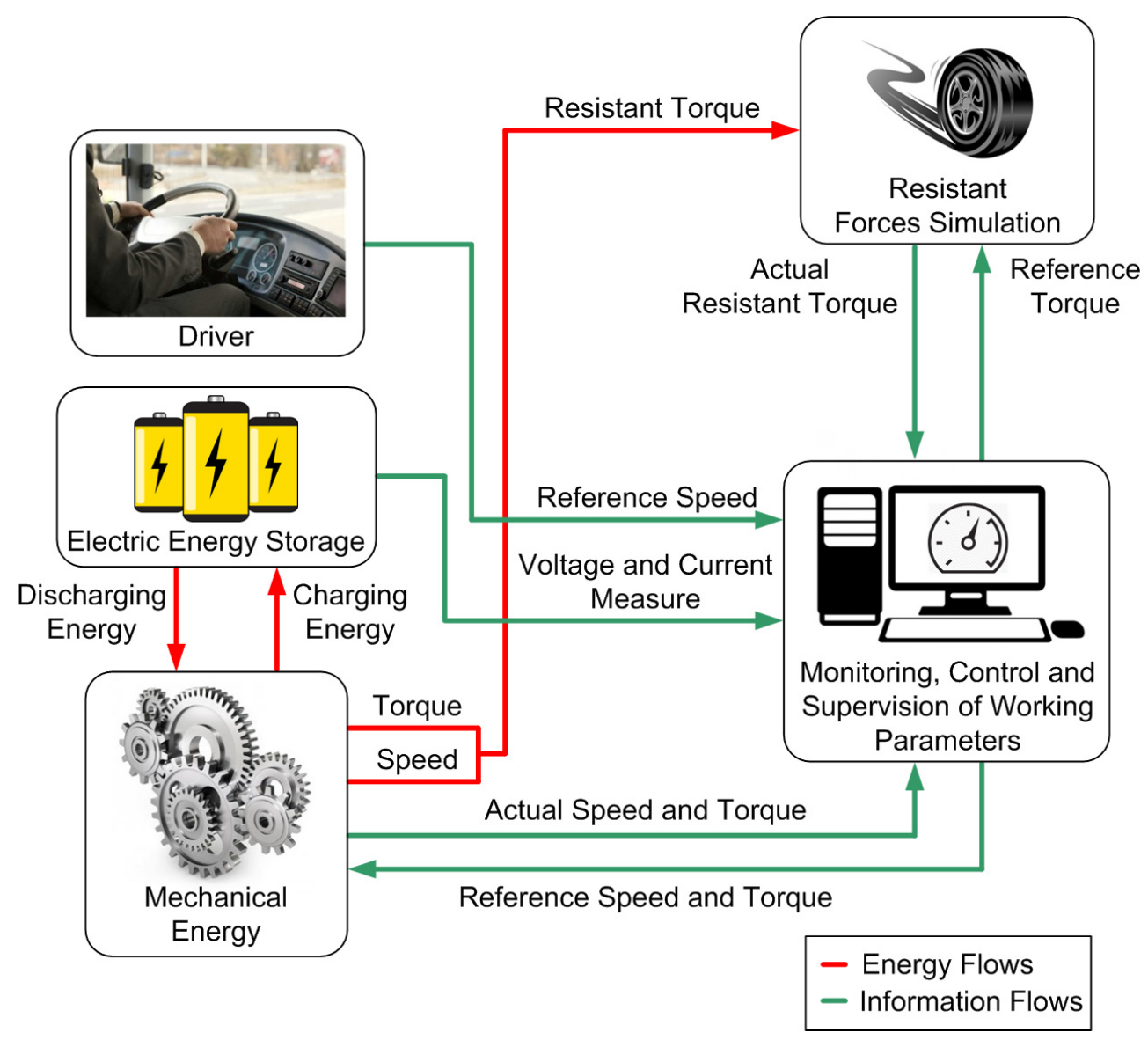

Fig. 2. Functional map of the propulsion system for the urban electric busses.

test-bench, is required to have the main functionalities reported as follows:

- supplying and storing the electric energy required by the electric drive;

- supplying the vehicle with the mechanical energy required by the driving cycle;

- simulating the driver in order to follow the reference speed of the driving cycle;

- simulating resistant forces related to vehicle and road parameters;

- monitoring and controlling electrical and mechanical parameters.

The functionalities reported above are interconnected through red and green arrows, which respectively represent energy and information flows.

The logical analysis, which represents the third step of the RFLP approach, aims to evaluate the electrical and mechanical performance of the system under study. The modelling operations of this phase are preliminarily carried out taking advantage of the software Dymola, which is based on the Modelica environment and is specifically devoted to the simulation of complex and multiphysical systems. Then, the system logical model is integrated into CATIA V6 by means of the dynamic behaviour modeling (DBM) workbench provided by Dassault Systemes [17]. Each component of the propulsion system is simulated
Table 2. Main characteristics and operative conditions of a single $\mathrm{Li}(\mathrm{NiCoMn}) \mathrm{O}_{2}$ cell.

\begin{tabular}{cc}
\hline Rated voltage (V) & 3.65 \\
Recommended minimum & 3.0 \\
Discharging voltage (V) & 4.15 \\
Maximum charging voltage $(\mathrm{V})$ & 174 \\
Specific energy (Wh.Kg ${ }^{-1}$ ) & 370 \\
Energy density $\left(\mathrm{Wh}^{-1}\right)^{-1}$ & $217 \times 129 \times 7.2$ \\
\hline Cell dimensions $(\mathrm{mm})$ & \\
\hline
\end{tabular}

through simplified models, mainly based on the information reported both on the datasheets and in literature. In fact, the main aim of this paper is not devoted to the modelling of specific components.

The first component, which is taken into account in the logical analysis of the whole power train, is the energy storage system. On the base of the above hypothesis the model of the vehicle battery pack is simulated with the static resistance single-stack model [18], through the 'Electric Energy Storage' Modelica library. The input electrical parameters of the above model are related to the case of a $\mathrm{Li}[\mathrm{NiCoMn}] \mathrm{O}_{2}$ cathode based battery pack. In fact, this battery technology has been widely investigated in the scientific literature for its good performance in supplying full electric and hybrid road vehicles [2-4]. The main characteristics and operative conditions for a single cell of the above battery pack are reported in Table 2 [19]. 


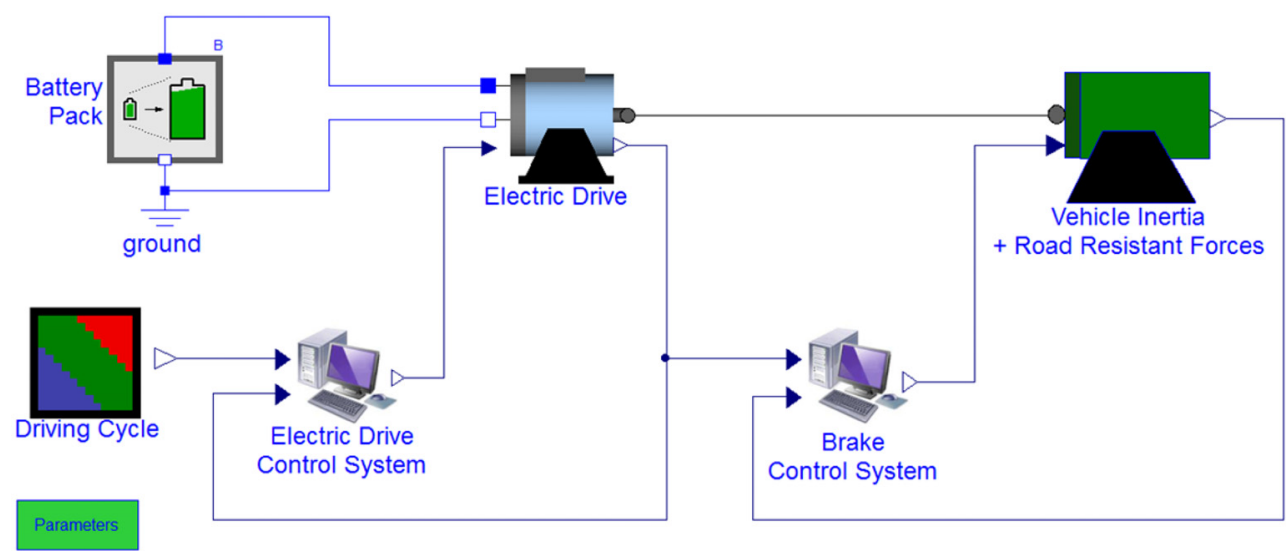

Fig. 3. Logical model of the propulsion system of the urban electric busses running on the test-bench.

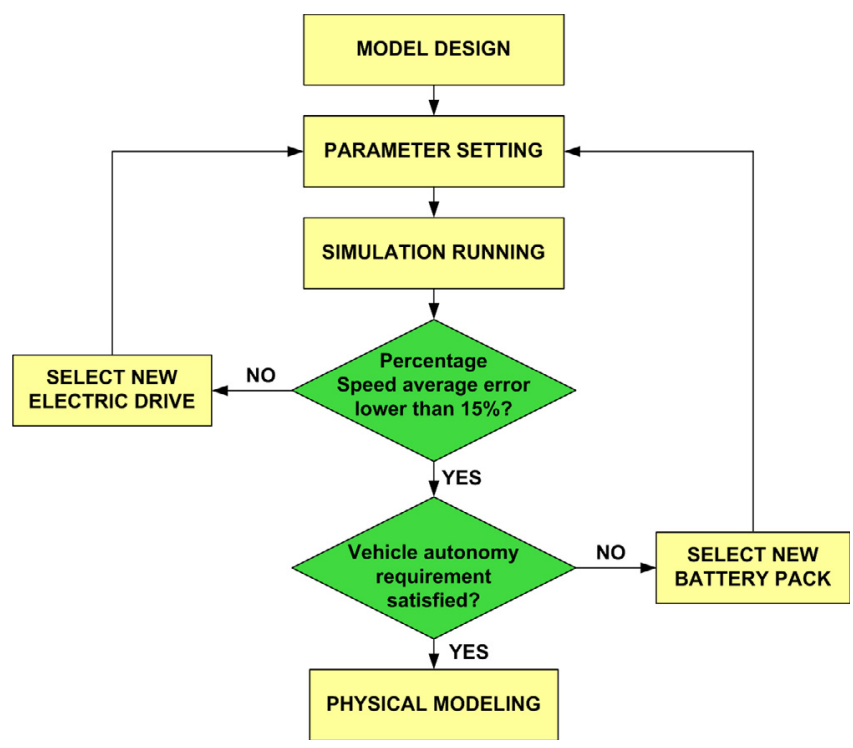

Fig. 4. Flow-chart of the design verification procedure.

The electric drive, in the same way as the battery pack, is simulated by means of specific blocks, available in the Modelica library, taking into account the rated and maximum torque of the electric motor as a function of the rotor angular speed. The propulsion system is considered directly connected, in the model, to an inertia block simulating the vehicle inertia and road resistance forces. The dynamic brake model is realized using the Dymola mechanical libraries. In particular, the vehicle inertia and road resistance forces are evaluated trough Modelica scripts and reported to the brake rotor shaft. The complete logical block scheme of the propulsion system running on the test-bench is reported in Figure 3.

At the end of the Dymola modelling phase, preliminary simulations are performed in order to analyse the behaviour of the whole system running on specific driving cycles. At this point, it is possible to verify the right design of the whole power train, following a specific verification procedure. The flow-chart related to the verification procedure is reported in Figure 4.
As described in Figure 4, after the definition of the logical model, the main simulation parameters are required to be set, taking into account the specific case study. At this point it is possible to run the simulations and evaluate the related numerical results, in order to verify the designing process through the presented iterative procedure. In fact, the first evaluation is based on the comparison between the actual and the reference vehicle speed, which is imposed by the specific driving cycle. This evaluation is aimed to analyse the performance of the propulsion system in terms of speed, when the vehicle is running on a specific road. If the average percentage error between the actual and reference speed is higher than $15 \%$, the electric drive is not considered suitable to satisfy the dynamic requirements of the driving cycle and a new electric drive is selected from a database. This database has been preliminarily populated with the main characteristics of a large number of electric drives with different rated power and speed. The second evaluation block is related to the vehicle autonomy, expressed in terms of hours, on the considered driving cycle. In particular, for the analysed case study, the autonomy is required to be greater than $4 \mathrm{~h}$, when the vehicle is running on a plain road, and greater than $2 \mathrm{~h}$ with a road slope of $10 \%$. In all the test analysed, the simulations are stopped when the vehicle battery pack reaches the State of Charge of 20\%, which represents the minimum SoC to avoid the reduction of battery pack durability. If the autonomy requirements are not satisfied, a $\mathrm{Li}[\mathrm{NiCoMn}] \mathrm{O}_{2}$ battery pack of greater capacity is chosen and the simulation runs again, taking into account the increase in vehicle weight and size related to the new battery pack. The values of size and weight for the new battery pack are evaluated taking into account the volumetric and gravimetric energy density of the considered battery technology.

When the propulsion system under study satisfies speed and autonomy requirements, the above iterative procedure ends and the designing process can be considered as optimized. At this point the evaluated dimensions of the optimized propulsion systems can be used for the physical modelling by means of the parametric 3D CAD model realized in CATIA V6 environment. 


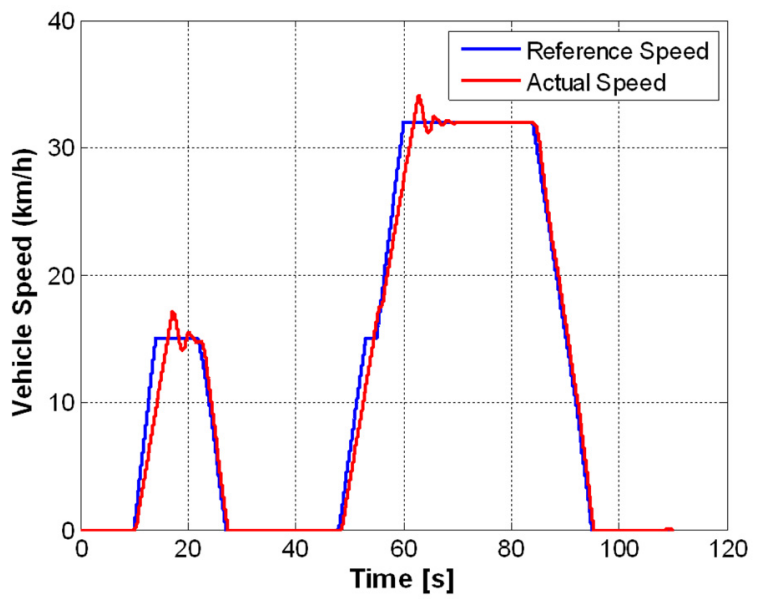

Fig. 5. Comparison between the actual and reference vehicle speed on the considered driving cycle for the first configuration.

\section{Simulation results and discussion}

The simulations carried out in this paper are aimed to verify the designing process of the propulsion system under study, starting from an analysis of its performance, when supplying the reference vehicle in different operative conditions. For the analysis reported in this section the first two steps of the ECE 15 driving cycle is considered [18].

Following the verification procedure reported in Figure 4 , the first set of simulations is based on the parameters of first configuration, which consists in a battery pack of $50 \mathrm{Ah}$ and electric drive of $54.6 \mathrm{~kW}$.

Figure 5 reports, for the above configuration, the comparison between the actual and the reference vehicle speed running on the considered driving cycle on a plain road.

The above comparison shows that this first configuration is not able to satisfy the dynamic requirement of the driving cycle. In fact an average percentage error between reference and actual vehicle speed of about 17\% is evaluated. This error is justified by the low value of motor torque, in comparison with vehicle inertia and road resistant forces. For this reason, after different iterations of the procedure reported in Figure 4, a new configuration, characterized by a $63.4 \mathrm{~kW}$ electric drive and a $150 \mathrm{Ah}$ battery pack, is selected. Although the new parameters affect the vehicle weight, the dynamic requirements of the trapezoidal driving cycle on plain road are satisfied by the urban bus supplied by the considered propulsion system, with an average percentage speed error of about $13 \%$. For this test, Figure $6 \mathrm{~A}$ shows the comparison between electric and mechanical power reported to the motor shaft during the considered driving cycle. In this case, a maximum mechanical power of about $80 \mathrm{~kW}$ is evaluated at the end of acceleration phase, whereas the negative values of mechanical power are related to regenerative operations during the deceleration phases. As shown in Figure 6B, State of Charge of the battery pack decreases of $0.35 \%$ in $110 \mathrm{~s}$. This means that the minimum $\mathrm{SoC}$ of $20 \%$ is reached after about $6 \mathrm{~h}$. For this reason also the autonomy requirement of the verification procedure is satisfied by this last configuration.

Another set of simulations is carried out in order to analyse the behaviour of the propulsion system when the vehicle is running on the above driving cycle with a positive road slope of $10 \%$. According to the simulation results, the propulsion system, equipped with the last identified configuration, is not able to perform the new driving cycle characterized by a different road slope. For the above reasons, after few iterations of the design verification procedure, another configuration, characterized by $162 \mathrm{~kW} / 1400 \mathrm{Nm}$ electric motor with a 300 Ah battery pack, is selected. In this case, the vehicle follows the reference speed, with a percentage error value of about $6 \%$, although the vehicle weight is quite high. Figure 7A shows the vehicle mechanical power, reported to the motor shaft, and the battery electric power during the performance of the trapezoidal driving cycle with a slope of $10 \%$. In this case, a maximum value of electric power of about $190 \mathrm{~kW}$ is reached during the acceleration phase. As shown in Figure 7B, State of Charge of the battery pack decreases of about $0.8 \%$ in $110 \mathrm{~s}$. This means that the minimum SoC of $20 \%$ is reached after about $3 \mathrm{~h}$. For this reason this last configuration satisfies also the autonomy requirement.

The results of the simulation activity, carried out in this paper, show that a proper combination of components realizing an optimized configuration of the propulsion system for the reference vehicle can be identified on the base of the RFLP approach. In addition, the designing approach presented in this paper, enriched by means of parametric relations building the final test bench CAD model implemented in CATIA V6, represents a design procedure which is expected to achieve a drastic reduction of designing times and costs for the whole development process of propulsion systems for road electric vehicles.

\section{Conclusions}

This paper deals with a multi-domain modelling and verification procedure aimed to the preliminary design of a propulsion system for road electric vehicles. The MBSE approach for the collaborative design of complex systems is used, in particular adopting the derived RFLP method. A top-down approach is adopted in the present work. At the system level, in fact, the architecture of the system is firstly developed, by specifying the components, the interfaces and the connections. Then, the internal architecture of every component is detailed at the component level. To validate the developed model, the vehicle speed calculated through the developed models is compared to that of a standard driving cycle. Results show a good agreement between the inputs and calculated parameters.

The MBSE approach used in this paper allows designers to define the system functional architecture starting from the design requirements. The logical level models are used to define the possible solutions that can be used to develop the behaviour of the system. The Logical-Physical integration is illustrated by integrating Modelica language 
Table 3. Parameters of First Configuration of propulsion system for the Trapezoidal Driving Cycle 0\% Slope Simulation.

\begin{tabular}{cccccc}
\hline \multirow{2}{*}{ Battery Pack } & Cells in series & Cells in parallel & Cell capacity & Cell weight & Battery pack weight \\
\cline { 2 - 6 } & 140 & 1 & $50 \mathrm{Ah}$ & $1.25 \mathrm{~kg}$ & $175 \mathrm{~kg}$ \\
\hline \multirow{2}{*}{ Electric Drive } & Power & Maximum torque & Rated current & Rotor inertia & Weight \\
\cline { 2 - 5 } & $54.6 \mathrm{~kW}$ & $470 \mathrm{Nm}$ & $101 \mathrm{~A}$ & $0.123 \mathrm{~kg} \cdot \mathrm{m}^{-2}$ & $130 \mathrm{~kg}$ \\
\hline
\end{tabular}
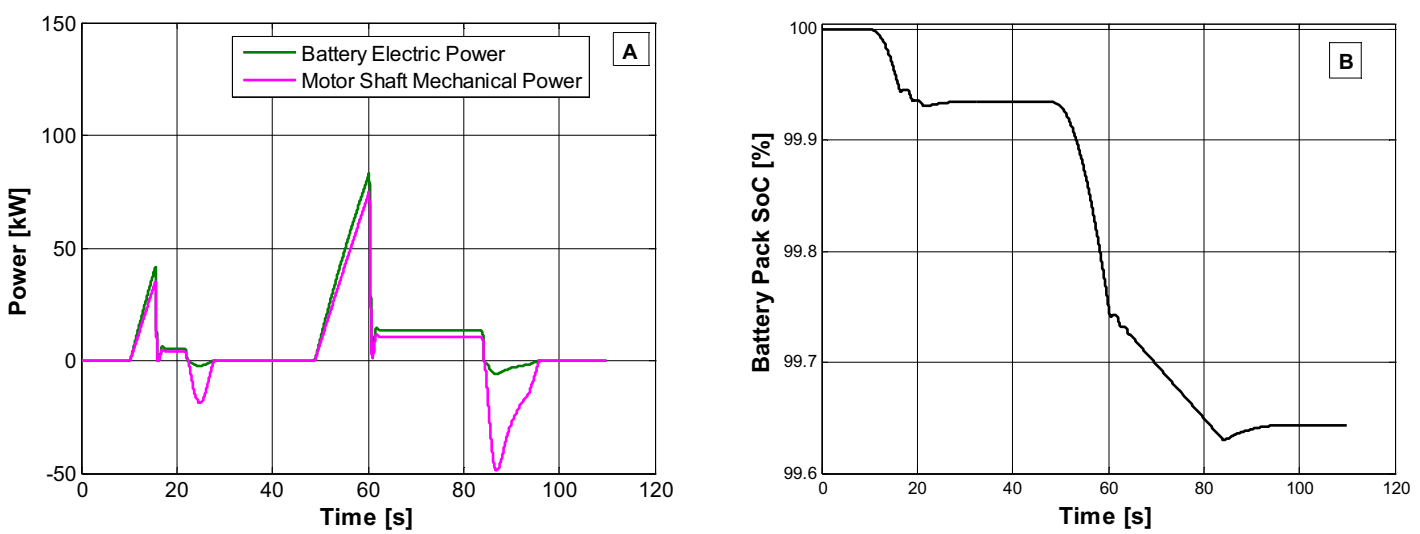

Fig. 6. Vehicle mechanical and electric power (A) reported to the motor shaft and battery SoC (B) vs time during the first two steps of the ECE15 driving cycle.
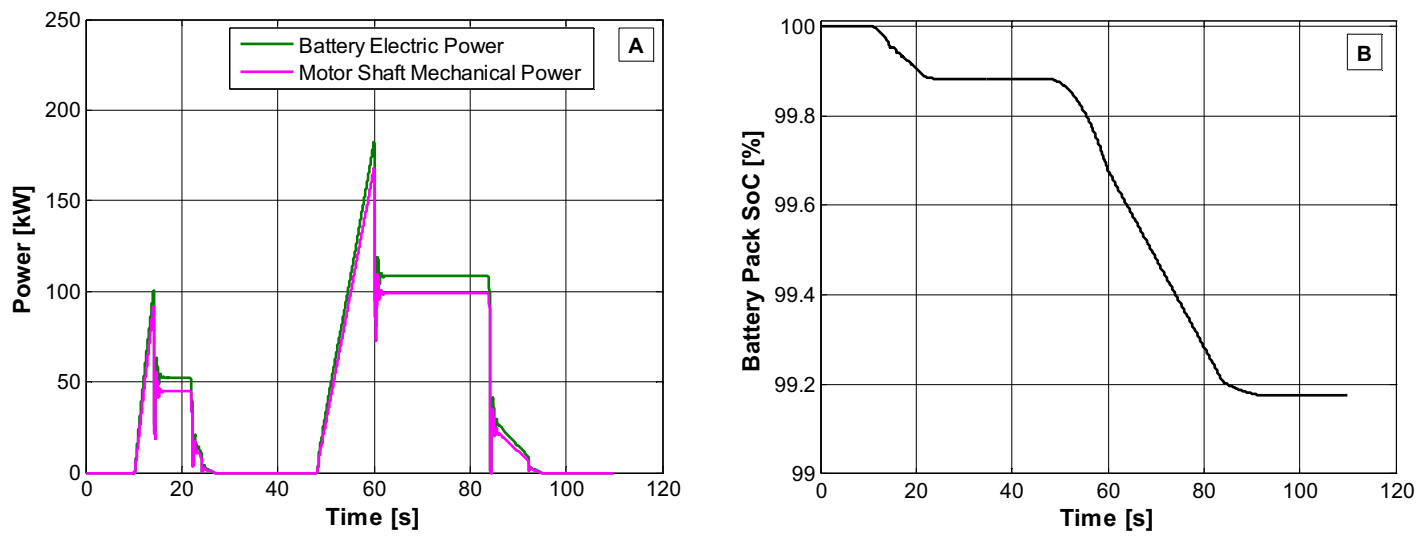

Fig. 7. Vehicle mechanical and electric power (A) reported to the motor shaft and battery SoC (B) vs. time during the first two steps of the ECE15 driving cycle.

models to every logical component. The interfaces between the logical components and their connectors are converted to Modelica interfaces and connectors. LogicalPhysical integration could also be illustrated by allocating $3 \mathrm{D}$ geometric representation to logical components.

Acknowledgements. The present work was developed with the contribution of the French Ministry for Higher Education performing the activities of the PLACIS (Plateforme Collaborative d'Ingénierie Système, Collaborative Platform for Systems Engineering) project. The authors gratefully acknowledge Mr. Antonio Rossi, technician of Istituto Motori, for his cooperation in the drawings of this paper.

\section{References}

[1] International Council on Systems Engineering, Systems engineering handbook: A guide for system life cycle processes and activities, edited by C. Haskins, International Council of Systems Engineering, 2008
[2] B. Gumus, A. Ertas, D. Tate, I. Cicek, Transdisciplinary Product Development Lifecycle, J. Eng. Design 19 (2008) $185-200$

[3] P. Graignic, T. Vosgien, M. Jankovic, V. Tuloup, J. Berque, N. Troussier, Complex System Simulation: Proposition of a MBSE Framework for Design-Analysis Integration, Proc. Comput. Sci. 16 (2013) 59-68

[4] M. Hammadi, J. Choley, O. Penas, A. Riviere, Multidisciplinary approach for modelling and optimization of Road Electric Vehicles in conceptual design level, Proc. of Electrical Systems for Aircraft, Railway and Ship Propulsion, 2012

[5] C. Zheng, M. Bricogne, J. Le Duigou, B. Eynard, Survey on mechatronic engineering: a focus on design methods and product models, Adv. Eng. Inform. 28 (2014) 241257

[6] P. Hehenberger, F. Poltschak, K. Zeman, W. Amrhein, Hierarchical design models in the mechatronic product development process of synchronous machines, Mechatronics 20 (2010) 864-875 
[7] F. Mhenni, J.-Y. Choley, O. Penas, R. Plateaux, M. Hammadi, A SysML-based methodology for mechatronic systems architectural design, Adv. Eng. Inform. 28 (2014) 218-231

[8] R. Mordinyi, D. Winkler, F. Waltersdorfer, S. Scheiber, S. Biffl, Integrating Heterogeneous Engineering Tools and Data Models: A Roadmap for Developing Engineering System Architecture Variants, In "Software Quality. Software and Systems Quality in Distributed and Mobile Environments", Springer, 2015, pp. 89-107

[9] P. Franciosa, S. Patalano, A. Riviere, 3D Tolerance Specification: an Approach for the Analysis of the Global Consistency based on Graphs, Int. J. Interactive Design Manuf. 4 (2010) 1-10

[10] G. Di Gironimo, C. Di Martino, A. Lanzotti, A. Marzano, G. Russo, Improving MTM-UAS to predetermine automotive maintenance times, Int. J. Interactive Design Manuf. 6 (2012) 265-273

[11] C. Capasso, M. Hammadi, S. Patalano, R. Renaud, O. Veneri, RFLP Approach in the Designing of Power-Trains for Road Electric Vehicles, In Multiphysics Modelling and Simulation for Systems Design and Monitoring, Springer International Publishing, pp. 249-258

[12] Y. Ni, J.F. Broenink, A co-modelling method for solving incompatibilities during co-design of mechatronic devices, Adv. Eng. Inform. 28 (2014) 232-240

[13] S. Kleiner, C. Kramer, Model Based Design with Systems Engineering Based on RFLP Using V6, Proc. of the 23rd CIRP Design Conference, 2013, DOI: 10.1007/978-3-642-30817

[14] A. Lanzotti, S. Patalano, F. Vitolo, A Graph-based Software Tool for the CAD Modeling of Mechanical
Assemblies, Proc. of 8th International Conference on Computer Graphics, Theory and Applications, GRAPP 2013 IVAPP 2013, Barcelona, Spain, 21-24 February, SciTePress Digital Library, 2013, pp. 60-69, DOI: 10.5220/0004299000600069

[15] O. Veneri, F. Migliardini, C. Capasso, P. Corbo, Dynamic behaviour of Li batteries in hydrogen fuel cell power trains, J. Power Sources 196.21 (2011) 9081-9086

[16] P. Smolek, B. Heinzl, B. Ecker, B. Breitenecker, Exploring the Possibilities of Co-Simulation with CATIA V6 Dynamic Behavior Modeling, Proc. of Electrotechnical and Computer Science Conference ERK, 2013

[17] M. Einhorn, F.V. Conte, C. Kral, S.C. Nikla, A Modelica Library for Simulation of Electric Energy Storages. Proc. of the 8th International Modelica Conference, March 2022, Technical Univeristy, Dresden, Germany, 2011, DOI: 10.3384/ecp11063436

[18] O. Veneri, F. Migliardini, C. Capasso, P. Corbo, Experimental performance assessment of $\mathrm{Pb}$, $\mathrm{Li}[\mathrm{NiCoMn}] \mathrm{O}_{2}$ and $\mathrm{LiFePO}_{4}$ batteries for road vehicles. Proc. of Power Electronics, Electrical Drives, Automation and Motion (SPEEDAM), 2012, DOI: 10.1109/SPEEDAM.2012.6264626

[19] T.J. Barlow, S. Latham, I.S. Mccrae, P.G. Boulter, A reference book of driving cycles for use in the measurement of road vehicle emissions, TRL Published Project Report, 2009, ISSN 0968-4093

[20] P. Gwangmin, L. Seonghun, J. Sungho, K. Sangshin, Integrated modeling and analysis of dynamics for electric vehicle powertrains, Expert Syst. Appl. 41 (2014) 25952607 